\title{
Synthesis and Characterization of Aluminium Oxide $\left(\mathrm{Al}_{2} \mathrm{O}_{3}\right)$ Nanoparticles and its Application in Azodye Decolourisation
}

\author{
Vijaya Pandurang Dhawale ${ }^{1, *}$, VaideiBalraj Khobragade ${ }^{2}$, Satish Damodar Kulkarni ${ }^{2}$ \\ ${ }^{1}$ Post Graduate Department of Physics, New Arts Commerce and Science College, Parner, Ahmednagar, India \\ ${ }^{2}$ Post Graduate Department of Environmental Science, New Arts Commerce and Science College, Ahmednagar, India \\ Email address: \\ vijaya.dhawale2009@gmail.com(V.P.Dhawale) \\ ${ }^{*}$ Corresponding author
}

\section{To cite this article:}

Vijaya Pandurang Dhawale, Vaidei Balraj Khobragade, Satish Damodar Kulkarni. Synthesis and Characterization of Aluminium Oxide $\left(\mathrm{Al}_{2} \mathrm{O}_{3}\right)$ Nanoparticles and its Application in Azodye Decolourisation. International Journal of Environmental Chemistry.

Vol. 2, No. 1, 2018, pp. 10-17. doi: 10.11648/j.ijec.20180201.13

Received: March 29, 2018; Accepted: May 4, 2018; Published: May 30, 2018

\begin{abstract}
The adsorption behaviour of an azodye Methylene Blue (MB) over aluminum oxide nano particles (AONP) generated by sol-gel method has been studied to investigate the physicochemical process involved andexplore the potential use of AONP in wastewater treatment. The variables incorporated in the present study are concentration of dye, dosage of adsorbentand contact time. The characterizations of AONPs were carried out using X-ray diffractometry (XRD), Scanning Electron Microscopy (SEM), Fourier Transform Infrared Spectroscopy (FTIR), Energy Dispersive Analysis of X-rays (EDAX) and Raman spectroscopy. SEM image showed the distribution pattern of nanoparticles. FTIR spectra revealed thatfunctional groups (O-Al-O) are present. Raman spectra showed the crystalline nature of nanoparticles. Averagesize of $\mathrm{Al}_{2} \mathrm{O}_{3}$ nanoparticle from XRD peak was found to be $25 \mathrm{~nm}$ having rhombohedral structure. Chemical composition of AONPs was confirmed from EDAXspectroscopy measurement. The smaller dosage of AONP was tested for the photocatalytic degradation.
\end{abstract}

Keywords: Colour Pigment, Adsorbent, Adsorption, Decolourisation, Sol-gel Method

\section{Introduction}

In recent years, the use of nanotechnology has gained significant attention in environmental applications for wastewater treatment [1]. Nanoparticles in water treatment have been effectively utilized due to their unique characteristics such ashigh surface area to volume ratio, small size, availability of large number of reactive sites, and high capacity for regeneration [2]. The current water pollution problems, including water quality, canbeimproved using nanoadsorbents, nanocatalysts, nanostructured catalyticmembranes, and many other products and processes resulting fromthe advancement of nanotechnology [3]. During the last few years alargenumber of nanomaterials have been synthesized and used as adsorbentstoremovethe pollutants from the wastewater [4]. Inorganic nanomaterials such as iron and aluiminiumbasednanoadsorbents were firstly investigated which may be due to low manufacturing cost and high decontamination efficiency [5-9]. AONP is one of the mostimportant and extensively used ceramic materials as adsorbent for water and wastewater treatment, catalystsor catalyst supports for chemical reactions, electrical insulators, structural composites for spacecraft, abrasive, thermal wear coatings and membrane applications [10-13]. Aluminium oxide nanoparticle is reported to exist in several phases [14].

It is well knownfactthat presence of dyes in wastewater is the most undesirable. Dyes are water soluble in nature and most suited in their action in aqueous medium. Nowadays industrialwastewateris the major environmental problem. Many industries like plastics, pharmaceuticals, textiles, leather, cosmetics, paints and varnishes etc. make use of dyes to colour their final products. Subsequently, the wastewater effluents generated from these industries are highly coloured and its disposal in water bodies causes damage to aquatic flora and fauna. These dyes reduce penetration of sunlight which affectthephotosynthetic activities of aquatic flora. Number of dyes are xenobiotic and non-biodegradable in 
nature [15]. The traditional technologies for wastewater treatment effectively work upon the removal of biological oxygen demand (BOD), but reduction of BOD is ineffective against colour removal. The combination of technologies like adsorption and dye degradation can provide solution to thisproblemdespite these processes are in laboratory stage of development [16]. Chemical oxidation of dyes is very successful for azo dyes as it can initiate the cleavage of azo bond. The problem of secondary pollution due to formation of oxidized amines and chlorine, in case of $\mathrm{NaOCl}$, is incredible [17]. Advanced non-noble metal catalyst for the oxygen reduction reaction (ORR) is $\mathrm{Ni} / \mathrm{Co}-\mathrm{MOF}$ nanoflakes because of its excellent oxygen reduction catalytic activity, good methanol tolerance and good durability [18].

Due to special properties of the nanomaterials, it attracts much more attention. It is a new functional material and most of atoms on the surface of the nanoparticles are unsaturated, therefore can easily bind with other atoms. Nanoparticles have high adsorption capacity, also the adsorption process rapid and the operation is simple. So, interest is growing in the application of nanoparticles as adsorbents [19]. Adsorption is a conventional technology of dye removal having very high efficiency and simple process. Activated carbon is a very efficient adsorbent used mainly for cationic and anionic dyes. Theuse of carbon as adsorbent in wastewater treatment have become impractical due to competitive adsorption of other organic molecules along with dye molecules, therefore it can be used at end of the treatment processmainly for colour removal. The nonconventional adsorbents are in application due to their easy availability. Adsorption technology isanon-destructive technology involving phase change from aqueous phase to solid surface immobilization. Some techniques involvingadsorptionalong with the catalytic degradation of dye found to be more advantageous [20]. The application of nanoparticles as adsorbents has become as an interesting area of research because of their small particle size and high surface area. The more active sites are also capable of interacting with pollutant species [21]. The application of AONP has been evaluated for the removal of hexavalent chromium from aqueous medium [22]. Also study indicates that adsorption capacity remains unchanged after regeneration of nanosizedadsorbent [23].

Methylene blue (MB) is one of the most commonly used dyes in different industries such as textiles, printing and rubber [24-26]. The effluents from these industries are a major source of environmental pollution. Not only the water bodies become coloured, but there isdecrease in the dissolved oxygen of water and blocking of the sunlight, thereby disturbing the natural growth activity of aquatic life. Therefore, treatment of effluents containing dyes is one of the challenging problems in the field of environmental chemistry [27-31].

In the present study aluminium oxide nanoparticles (AONPs)were prepared by sol-gel method and used for the adsorptive removal of azo dye Methylene Blue (MB) from aqueous medium using batch adsorption technique.

\section{Experimental}

\subsection{Synthesis of Aluminium Oxide Nanoparticles}

The nanoparticles were prepared by the sol-gel technology. All chemicals used were analytical grade. aluminium chloride, $\mathrm{AlCl}_{3}$ (Molychem), 25\% $\mathrm{NH}_{3}$ solution (Qualigen Fine Chemicals) and polyvinyl alcohol (PVA) (Modern Industries) were used as raw materials for the synthesis of aluminium oxide nanoparticles.

$0.1 \mathrm{M}$ alcoholic $\mathrm{AlCl}_{3}$ solution was prepared, followed by addition of $25 \%$ ammonia solution. The resulting solution turned to a white sol. This was followed by the addition of PVA $(0.5 \mathrm{M})$. The solution was stirred continuously using a magnetic stirrer until it became a transparent sticky gel. The gel was allowed to mature for 24 hours at room temperature. The resultant gel was heat treated at $100^{\circ} \mathrm{C}$ for 24 hours which ledtothe formation of light weight porous materials due to the enormous gas evolution. The dried gel was, then calcined at $1200^{\circ} \mathrm{C}$ for 4 hours and finally, the calcined powders were crushed using mortar and pestle to get the fine homogeneous dense powder [32]. Figure 1 shows chemical structure of methylene blue (MB).<smiles>CN(C)c1ccc2nc3ccc(N(C)C)cc3[s+]c2c1</smiles>

Figure 1. Chemical structure of methylene blue (MB).

\subsection{Photocatalytic Degradation Studies}

Photocatalytic activities of the as-synthesized powder were evaluated by depolarization of Methylene blue dye in aqueous solution. The experiments were carried out in the presence of UV light irradiation without any adsorbent (blank), with adsorbent in daylight and in the presence of UV light with adsorbent. The photocatalytic reactor consists of a glass chamber with an inlet tube for provision of dye solution purging during photo catalysis and outlet tube for the collection of samples. Experimental arrangement is shown in figure 2.

Distilled water was used to prepare all stock solution and stored in dark bottles. From a stock solution of $1000 \mathrm{mg} / \mathrm{L}$ of the dye, different concentrations were made. Reaction was set up by adding different dose of the as-synthesized aluminium oxide nanopowder $(10,20,30 \mathrm{mg})$ into $1000 \mathrm{~mL}$ of MB solution of varying concentration (10, 15, 20, $25 \mathrm{mg} / \mathrm{L}$ ) in the glass chamber. The solution was magnetically stirred in with and without nanoparticle additioninto dye solution for 180 minute to obtain adsorptionequilibriumafter irradiating the internal and external UVlight sources in the chamber of the reactor. Sample was withdrawn at 60 minute time interval over contact time for 180 minute. The solution was centrifuged at $3000 \mathrm{rpm}$ for 10 minute and filtered 
through Whatman filter paperto remove the nano particles before measuring absorbance.

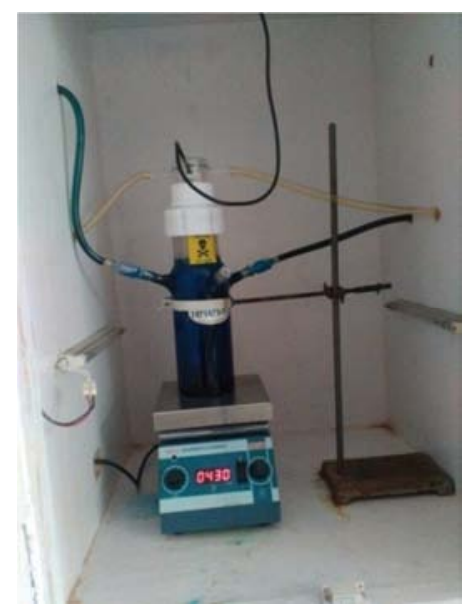

Figure 2. Experimental arrangement.

he absorbance of sample was measured at $580 \mathrm{~nm}$ using visible double beam spectrophotometer (Systronics, Model1203). For quantitative analysis percentage degradation of $\mathrm{MB}$ dye was calculated using the following formula:\% decolourisation $=\left(\mathrm{A}_{\mathrm{o}}-\mathrm{A}_{\mathrm{t}}\right) / \mathrm{A}_{\mathrm{o}} * 100$.

Where $A_{0}$ is absorbance of dye at initial stage, $A_{t}$ is absorbance of dye at time $t$.

$\mathrm{q}_{\mathrm{e}}=\left(\left(\mathrm{A}_{\mathrm{o}}-\mathrm{A}_{\mathrm{t}}\right) / \mathrm{W}\right) * \mathrm{~V}$

where qe is the amount of dye adsorbed at the time of equilibrium $(\mathrm{mg} / \mathrm{g}), \quad \mathrm{A}_{0}$ and $\mathrm{A}_{\mathrm{t}}$ are initial and final concentration of dye $(\mathrm{mg} / \mathrm{L}), \mathrm{V}(\mathrm{L})$ is the volume of sample, and $\mathrm{W}(\mathrm{g})$ is the mass of adsorbent $[33,41]$.

\section{Results and Discussion}

\subsection{Photocatalytic Degradation Study}

The photocatalytic activity of as-synthesized nanomaterial was evaluated by the degradation of $\mathrm{MB}$ dye in aqueous solution. The decolourisation of the MB dye was examined under three different conditions (treatments): daylight irradiation with and without nanopowder (blank), in presence of UVlight irradiation without nanopowder (in dark) and in the presence of $\mathrm{Al}_{2} \mathrm{O}_{3}$ nanopowder as aadsorbent under UVlight irradiation, respectively. For dark experiments (in the absence of the adsorbent) under UV light irradiation, almost insignificant degradation of the dye was observed.

In the absence of adsorbent (AONP), but in presence of UV light irradiation, average $10 \%$ decolourisation efficiency was observed throughout the $180 \mathrm{~min}$ reaction time. This result confirms that degradation of MB in the absence of the adsorbent, but presence of UVlight irradiation is insignificant. Further, the experimental results showed that when the dye solution is exposed to UVlight irradiation for $180 \mathrm{~min}$ in the presence of AONPasa adsorbent, significant degradation ofMB dye was observed. The corresponding plots of percentremovalof MB dye as function of time under UVirradiation in the presence and absence of AONP adsorbent are as shown in figure3 to figure6. Accordingly, the degradation efficiency of MB dye under the UV light was found to be much larger than the degradation efficiency as compared to dark treatment.

The degradation efficiency of AONP in the presence of UV light increased due to the fact that the $\mathrm{Al}_{2} \mathrm{O}_{3}$ nanoparticles prepared by the sol gel method has a high specific surface area, that could give more active surface sites to absorb water molecules and to form active $\mathrm{HOOandOH}$ radicals by trapping the photo generated holes.

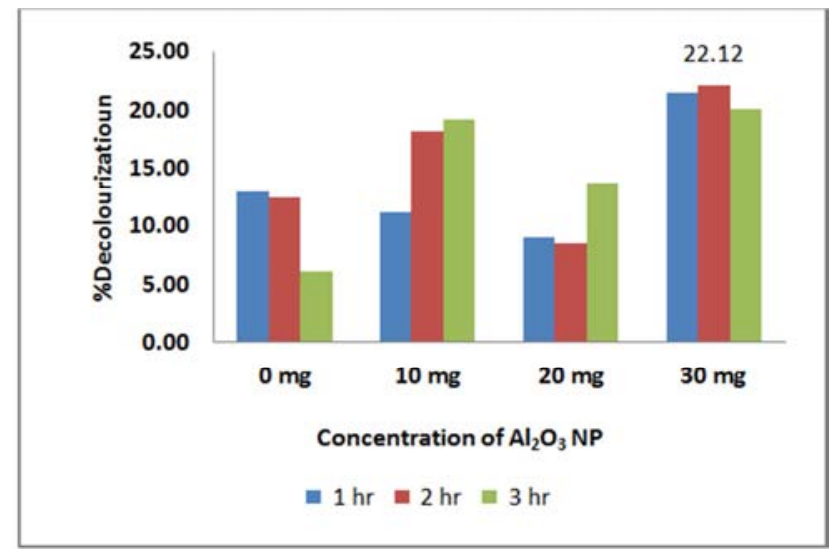

Figure 3. Decolourization of methylene blue Dye (10 mg) using $\mathrm{Al}_{2} \mathrm{O}_{3}$ Nanoparticles.

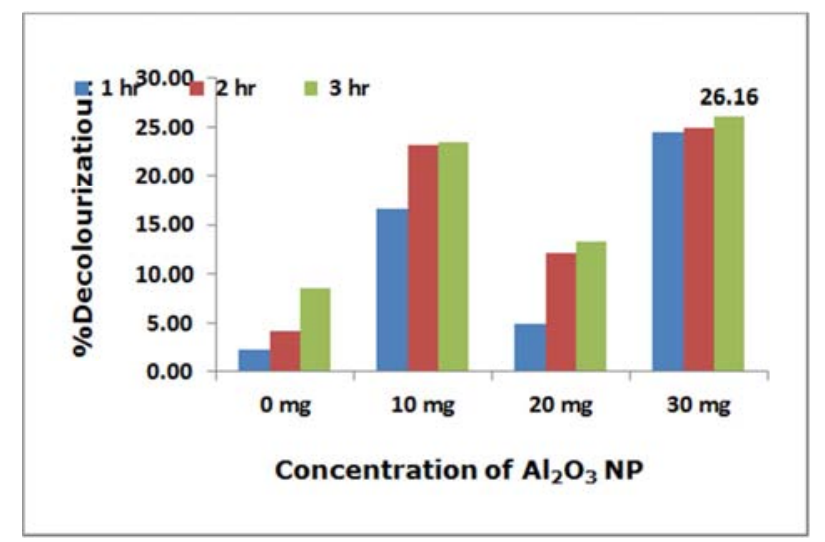

Figure 4. Decolourization of methylene blue dye (15 mg) using $\mathrm{Al}_{2} \mathrm{O}_{3}$ Nanoparticles.

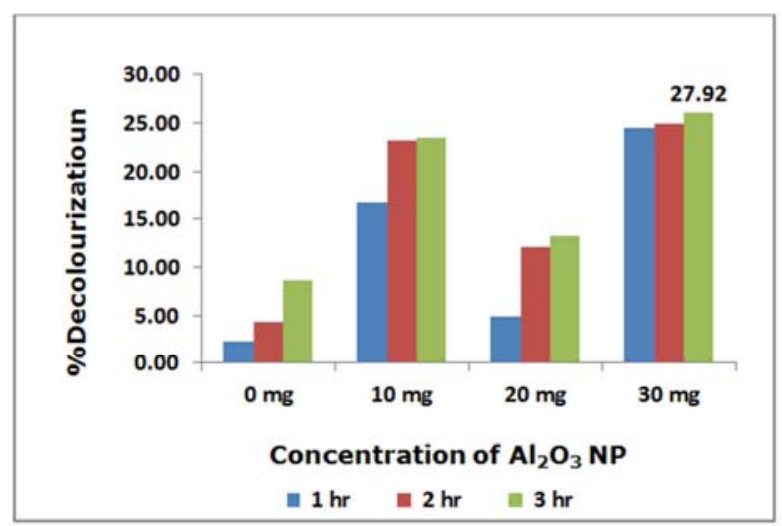

Figure 5. Decolourization of methylene blue dye (20 mg) using $\mathrm{Al}_{2} \mathrm{O}_{3}$ Nanoparticles. 


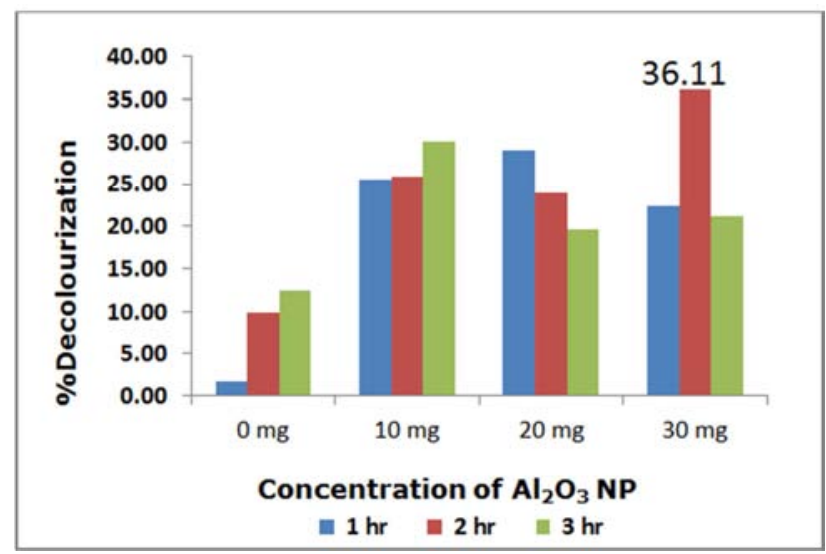

Figure 6. Decolourization of methylene blue dye (25 mg) using $\mathrm{Al}_{2} \mathrm{O}_{3}$ Nanoparticles.

Furthermore, these free active radicals drive the photo degradation reactions and leads to the decomposition of organic pollutants in the aqueous solution [31]. High surface area also facilitates the absorption of dye molecules on the surface of AONP. Under UVlight irradiation, MB molecules get absorbed on the surface of nanoparticlesand produce electrons. These electrons are captured by the surface adsorbed $\mathrm{O}_{2}$ molecules to yield $\mathrm{HO}_{2} \bullet$ and $\mathrm{O}_{2} \bullet-$ radicals, which gets more chance to come in contact with dye molecules and thus increasing the reaction speed.

The MB molecules thus could be mineralized in time by the super oxide radical ions. Thus it can be said that the smaller crystalline size of nanoparticle are favorable for the reduction of $\mathrm{O}_{2}$ and oxidation of $\mathrm{H}_{2} \mathrm{O}$ molecules by trapping electrons and holes, which improves the photocatalytic activity of the adsorbentunderUV light. The average amount of dye absorbed (\%qe) in batch experiment is $23.9 \mathrm{mg} / \mathrm{g}$ after 3 hours (Table 1).

Table 1. Amount of dye absorbed (\%qe) in batch experiment.

\begin{tabular}{lllll}
\hline Amount of adsorbent (AONP) & \multicolumn{3}{l}{ Concentration of Methylene Blue(MB) Dye } \\
\hline & $10 \mathrm{mg}$ & $15 \mathrm{mg}$ & $20 \mathrm{mg}$ & $25 \mathrm{mg}$ \\
$10 \mathrm{mg}$ & 18.7 & 34.2 & 35.4 & 69.9 \\
$20 \mathrm{mg}$ & 5.7 & 7.8 & 26.1 & 31.1 \\
$30 \mathrm{mg}$ & 9.0 & 14.1 & 12.8 & 22.0 \\
Total amount of dye absorbed at each concentration of dye after 3 hours & 33.4 & 56 & 74.3 & 122.9 \\
& Average amount of dye absorbed after 3 hours (\%qe) in & 23.9 \\
\hline
\end{tabular}

\subsection{Effect of Initial Methylene Blue (MB) Concentrations}

Effect of initial dye concentration on the degradation efficiency was investigated by varying initial dye concentration. The photo catalytic activity of the AONP is as shown in figure 3 to figure 7 . It was found that, photo degradation efficiency increased with increased concentration of MB dye and the adsorbent after 180 minutecontacttime.

\subsection{Effect of Amount of Adsorbent}

The amount of adsorbentalso affects photo degradation efficiency of the adsorbent. Different amounts of adsorbent wereused (10mg to $30 \mathrm{mg}$ ) for degradation of MB dye under UV light irradiation and the results are as shown in figure 3 to figure 7 . It was observed that, as the quantity of adsorbent increased, the rate of photo degradation of dye also increased. This may be due to the fact that, as the amount of adsorbent increases, the number of active sites on the adsorbent and the available surface area also increases. It should be noted that when the concentration of the adsorbent increases above the optimum value, the degradation rate decreases due to the interception of the light by the suspension [34, 35]. The excess adsorbent prevent the illumination thereby a primary oxidant, $\mathrm{OH}$ radical, in the adsorbentsystemalsodecreases and the efficiency of the degradation reduces accordingly [36]. Also, the increase of adsorbent concentration beyond the optimum value may result in the agglomeration of adsorbent particles.

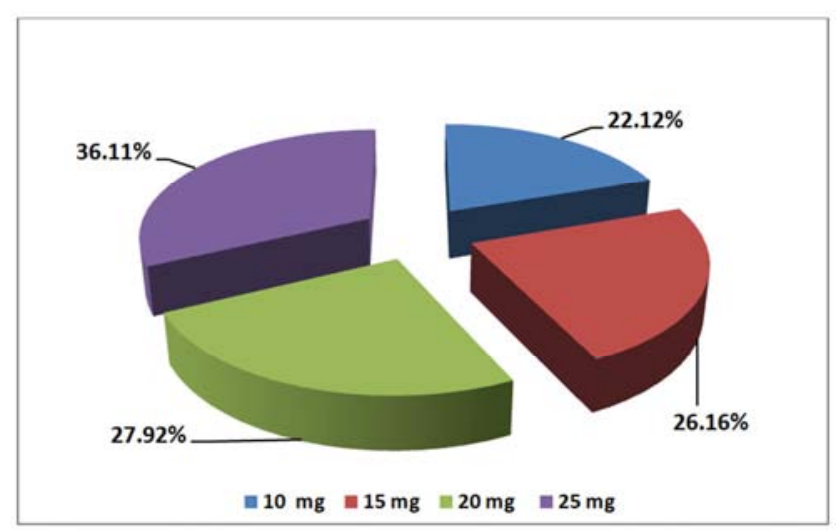

Figure 7. \% Decolourisation of Methylene Blue Dye at Various Concentrations Using $\mathrm{Al}_{2} \mathrm{O}_{3}$ Nanoparticles.

Therefore moreadsorbent surface become unavailable for photo absorption, thus resulting decrease in the degradation rate of adsorbent [37]. Beyond a certain limit of adsorbentamount, the solution becomes turbid whichblocks UV radiation for the reaction to proceed and therefore percentage degradation starts decreasing [38, 39]. For all these above reasons optimization of factors affecting the degradation process becomes necessary. 


\subsection{Effect of Contact Time}

The effect of contact time was investigated in the batch experiment at 10,15, 20 and $25 \mathrm{mg} / \mathrm{L}$ dye concentration. The adsorption capacity of dye showed varying results with increasing contact time. The rate of dye removal was initially high due to high concentration gradient and more availability of adsorption sites. The rapid transport of dye molecules from aqueous solution to the bulk makes the adsorption fast. The surface of AONPs is charged and the net charge at the surface at a particular $\mathrm{pH}$ governs the adsorptive removal of dye from the solution [15].

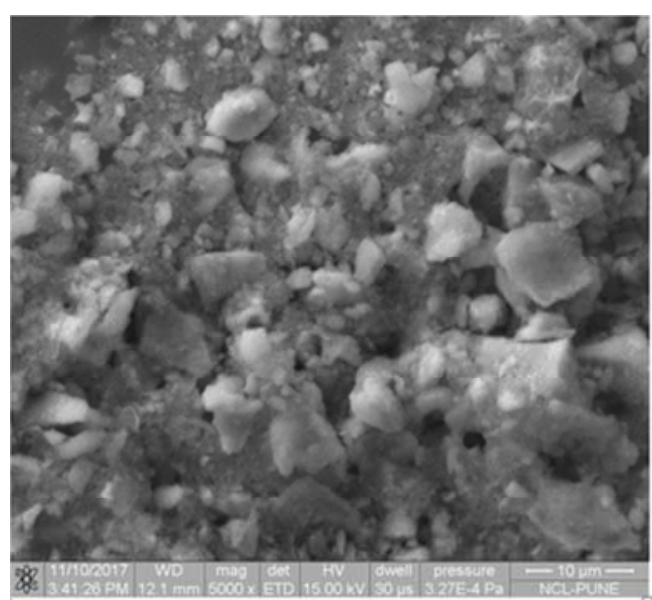

\subsection{Characterization}

The characterization of aluminium oxide nanoparticles were carried out using XRD, SEM, EDAX, FTIR, and Raman spectroscopy. SEM image gives the distribution pattern and size of the nanoparticles (Figure 8). TheFTIR spectra revealed that, there are 410.84, 420.48, 445.56, $491.85,501.49,588.29,636.51,709.80$ (O-Al-O) functional groups (Figure 9). Raman spectra shows the crystalline nature of the nanoparticles. In an amorphous state, the Raman bands are quite broad but often can be derived from one of the crystalline forms of the same material.

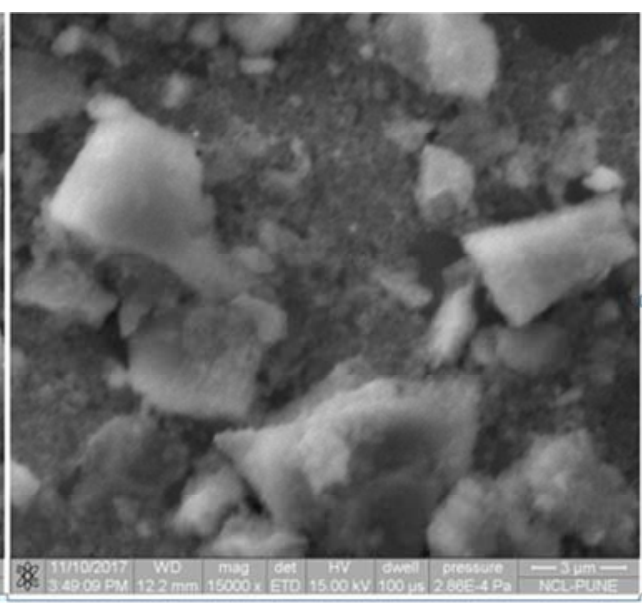

Figure 8. SEM of $\mathrm{Al}_{2} \mathrm{O}_{3}$ Nanoparticles.

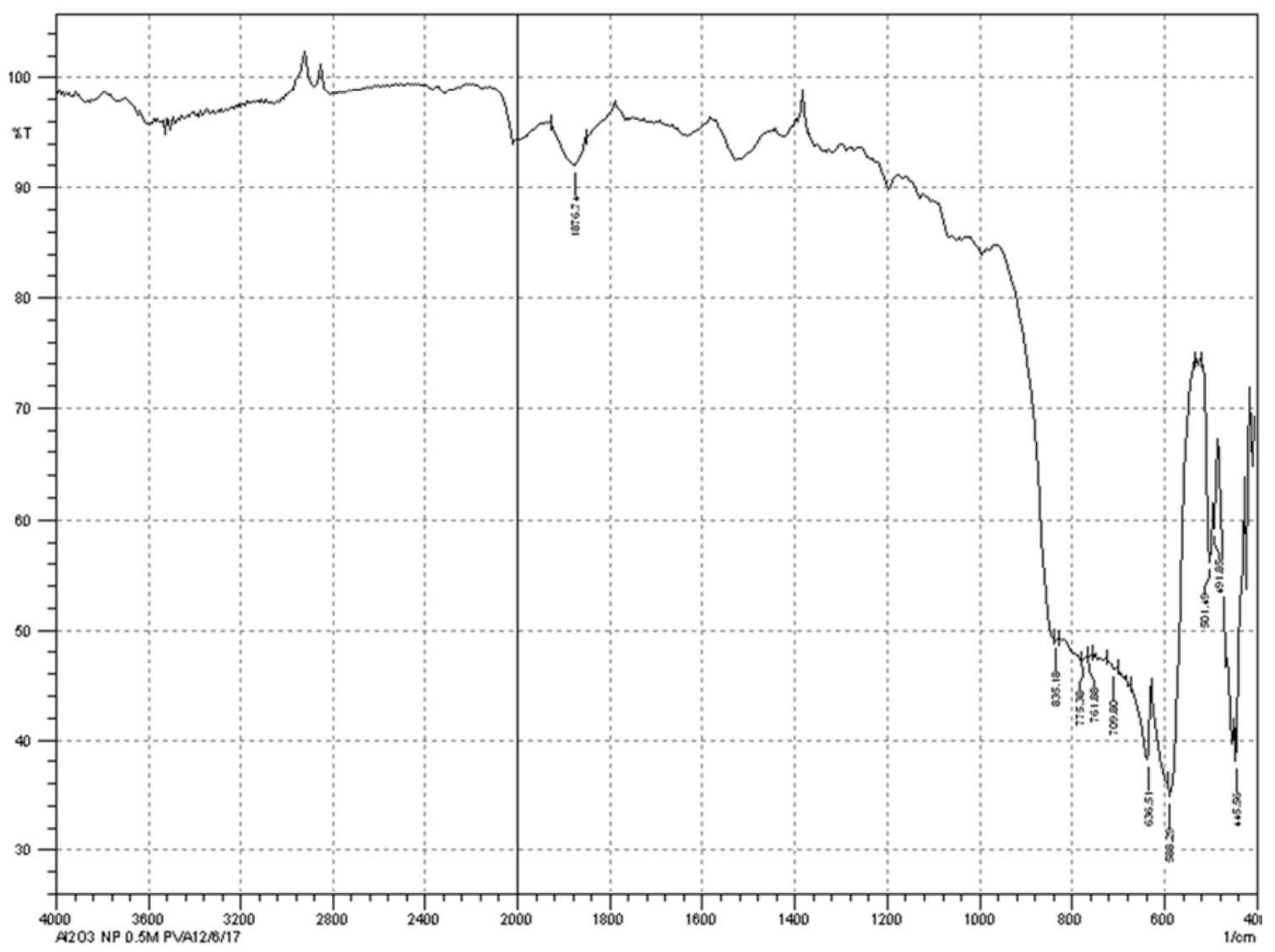

Figure 9. FTIR of $\mathrm{Al}_{2} \mathrm{O}_{3}$ Nanoparticles. 


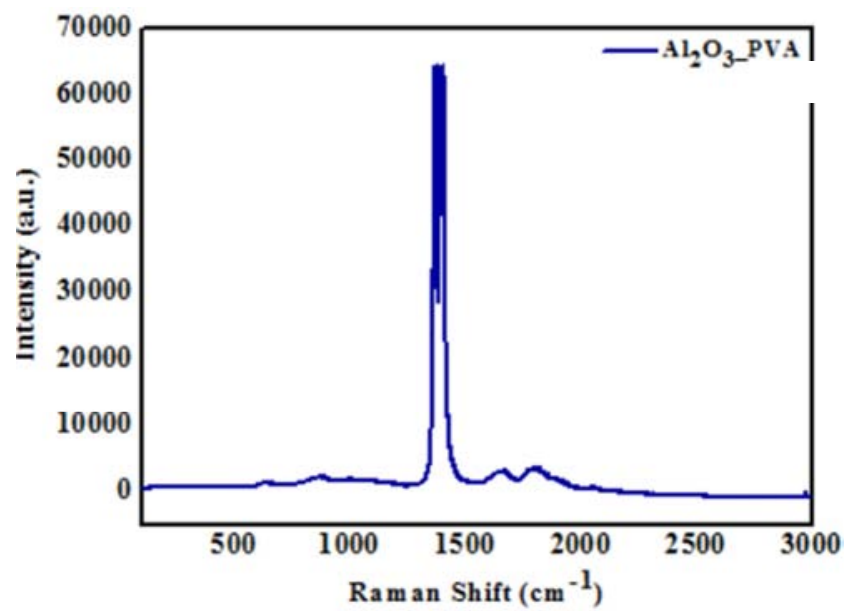

Figure 10. Raman Spectra of Al2O3 Nanoparticles.

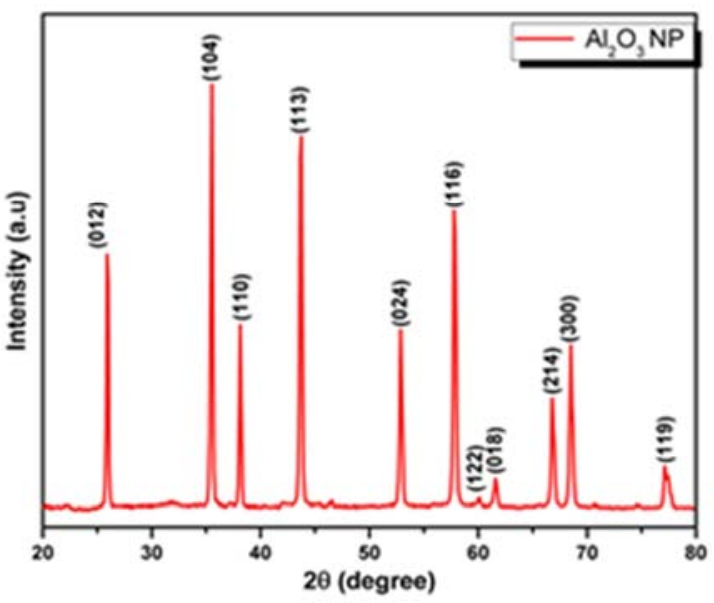

Figure 11. $\mathrm{XRD}$ of $\mathrm{Al}_{2} \mathrm{O}_{3}$ Nanoparticles.

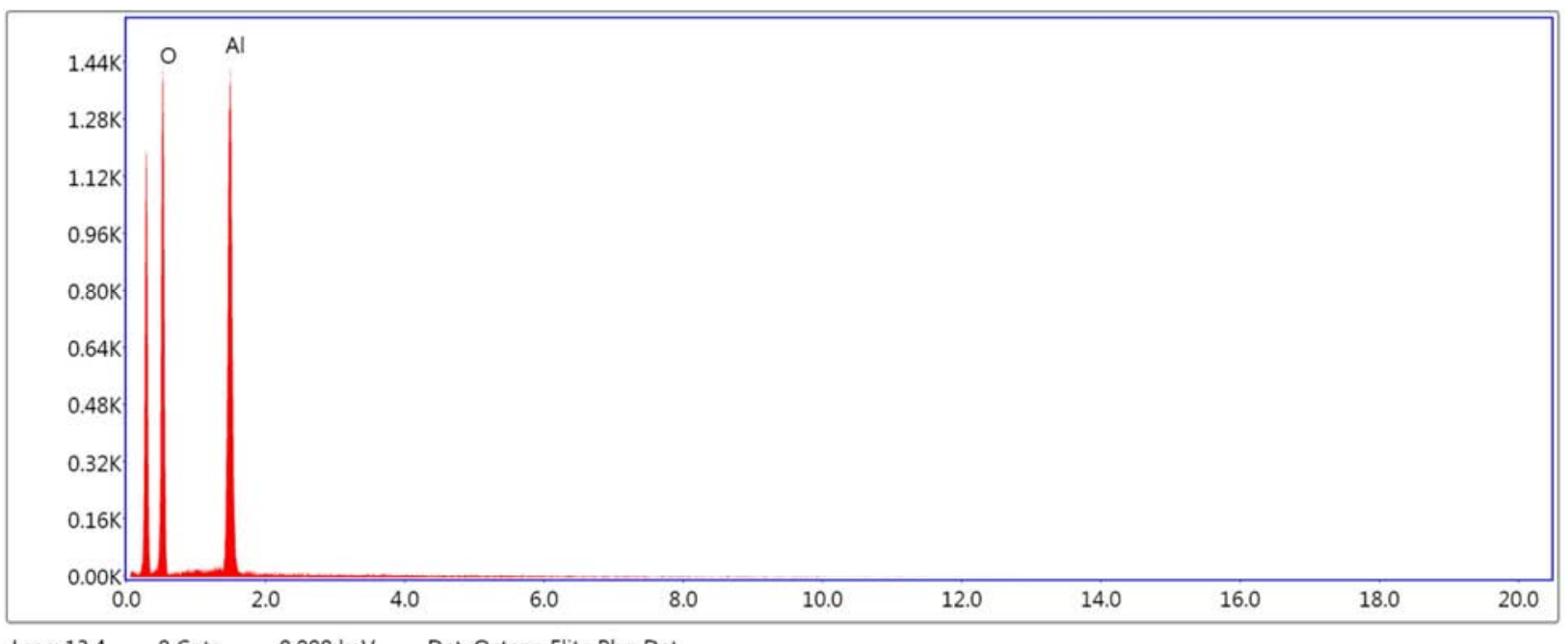

Lsec: $13.4 \quad 0$ Cnts $\quad 0.000 \mathrm{keV} \quad$ Det: Octane Elite Plus Det

Figure 12. EDAX of $\mathrm{Al}_{2} \mathrm{O}_{3}$ Nanoparticles.

In fact, in the case of ceramics, the transition from the amorphous to crystalline form (devitrification) can be an important phenomenon in terms of the performance of the ceramic.

The assumption is that in the amorphous form there is a distortion of interatomic bond angles. Long-range order is lost, but nearest neighbour interactions are affected so as to shift a particular vibrational frequency; because there is a population of such distortions, the observed band will be broad(Figure 10). Similar results have been obtained by others also [40]. The peaks in the XRD pattern significantly supportedformation of nanosized $\mathrm{Al}_{2} \mathrm{O}_{3}$ nanoparticles from JCPDS file (71-1683) having rhombohedralstructure. Nine reflections were observed at $2 \theta$ angles around $25^{\circ}(012)$, $35^{0}(104), 43^{0}(113), 52^{0}(024), 57^{0}(116), 61^{0}(122), 66^{0}(214)$, $68^{\circ}(300)$ and $70^{\circ}(119)$. Average particle size was calculated from all peaks and was found to be $25 \mathrm{~nm}$, whereas, the lattice constant 0.614 using Debye Scherrer formula, $\mathrm{D}=0.9 \lambda / \beta \cos \theta$ where, $\mathrm{D}$ is the crystalline size, $\lambda$ is the wavelength of $\mathrm{CuK} \alpha$ radiation, $\beta$ is full width half maximum (FWHM) of the diffraction peak and $\theta$ is Bragg's angle of X- ray diffraction peak (Figure 11). The quantitative analysis of the $\mathrm{Al}_{2} \mathrm{O}_{3}$ nanoparticle was done using EDAX spectroscopy measurement and it shows $\mathrm{Al}$ and Oas the major components of aluminiumoxide nanoparticlesin the heads as shown in figure 12 .

\section{Conclusions}

Aluminium oxide nanoparticles $\left(\mathrm{Al}_{2} \mathrm{O}_{3}\right)$ were successfully synthesizedvia sol gel technique and the average particle size was found to be $25 \mathrm{~nm}$ having rhombohedral structure. FTIR spectra of aluminium oxide nanoparticles indicated the formation of aluminium oxide nanoparticles $\left(\mathrm{Al}_{2} \mathrm{O}_{3}\right)$. X-Ray diffraction patterns confirm the formation of aluminium oxide $\left(\mathrm{Al}_{2} \mathrm{O}_{3}\right)$ nanoparticles. The formation of aluminium oxide $\left(\mathrm{Al}_{2} \mathrm{O}_{3}\right)$ nanoparticles was validated from Raman spectra, XRD, SEM and EDAX analysis.

Maximum decolourisation was found to be $36 \%$ for $25 \mathrm{mg}$ methylene blue (MB) dye concentration and $30 \mathrm{mg}$ dose of aluminium oxide (AONP) nanoparticles. The average amount of dye adsorbed in the batch experiment was $23.9 \mathrm{mg} / \mathrm{g}$. Thus, 
the above results support the recommendation that aluminium oxide nanoparticles offer new dimension towards reliable and economically affordable water treatment of coloured industrial effluents. The nanomaterial is verypromising and can be effectively used for the removal ofazodyes from the aqueous solutions.

\section{Acknowledgements}

Smt. Dhawale V. P. is thankful to University Grants Commission (WRO), Pune, India for providing the Teacher Fellowship. The authors are thankful to Dr. B. H. Zaware, Principal, New Arts, Commerce and Science College, Ahmednagar for providing necessary laboratory facilities.

\section{References}

[1] Brame, J., Li, Q., Alvarez, P. J. J., (2011). Nanotechnology enabled water treatment and reuse: emerging opportunities and challenges for developing countries. Trends Food Sci. Technol., 22: 618.

[2] Ali, I., (2012). New generation adsorbents for water treatment. Chem. Rev., 112: 5073.

[3] Savage, N., Diallo, M. S., (2005). Nanomaterials and water purification: opportunities and challenges. Jr. Nanoparticle Res., 7: 331.

[4] Mohmood, I., Lopes, C. B., Lopes, I., Ahmad, I., Duarte, A. C., Pereira, E., (2013). Nanoscale materials andtheir use in watercontaminants removal-a review. Environ. Sci. Pollut. Res., 20: 1239.

[5] Li, L., Fan, M., Brown, R. C., Leeuwen, J. V., Wang, J., Wang, W., Song, Y., Zhang, P., (2006). Synthesis, properties, and environmental applications of nanoscale iron-based materials: a review. Crit. Rev. Environ. Sci. Technol., 36: 405.

[6] Sharma, Y. C., Srivastava, V., Singh, V. K., Kaul, S. N., Weng, C. H., (2009). Nano-adsorbents for the removal of metallic pollutants from water and wastewater. Environ. Tech., 30: 583.

[7] Sharma, Y. C., Srivastava, V., Upadhyay, S. N., Weng, C. H., (2008). Alumina nanoparticles for the removal of Ni(II) from aqueous solutions. Ind. Eng. Chem. Res., 47: 8095.

[8] Hordern, B. K., (2004). Chemistry of alumina, reactions in aqueous solution and its application in water treatment. $A d v$. Colloid Interf. Sci., 110: 19.

[9] Giles, D. E., Mohapatra, M., Issa, T. B., Anand, S., Singh, P., (2011). Iron and aluminium based adsorption strategies for removing arsenic from water. Jr. Environ. Manage, 92: 3011.

[10] Sharma, Y. C., Srivastava, V., Mukherjee, A. K., (2010). Synthesis and application of nano- $\mathrm{Al}_{2} \mathrm{O}_{3}$ powder for the reclamation of hexavalent chromium from aqueous solutions. Jr. Chem. Eng. Data, 55: 2390.

[11] Srivastava, V., Weng, C. H., Singh, V. K., Sharma, Y. C., (2011). Adsorption of nickel Ions from aqueous solutions by nanoalumina: kinetic, mass transfer, and equilibrium studies. Jr. Chem. Eng. Data, 56: 1414.
[12] Yalamac, E., Trapani, A., Akkurt, S., (2014). Sintering and microstructural investigation of gamma-alpha alumina powders. Eng. Sci. Technol. Internat. Jr., 17: 2.

[13] Cai, W., Hu, Y., Yu, J., Wang, W., Zhou, J., Jaroniec, M., (2015). Template-free synthesis of hierarchical c- $\mathrm{Al}_{2} \mathrm{O}_{3}$ nanostructures and their adsorption affinity toward phenol and $\mathrm{CO}_{2}$. RSC Adv., 5: 7066 .

[14] Shek, C. H., Lai, J. K. L., Gu, T. S., Lin, G. M., (1997). Transformation evolution and infrared absorption spectra of amorphous and crystalline nano- $\mathrm{AI}_{2} \mathrm{O}_{3}$ powders. Nano Struct. Mater, 8: 605.

[15] Khosala, E., (2015). Adsorption of azo dye methyl orange overaluminum oxide nanoparticles. Internat. Jr. of Basic and Applied Chem. Sci., 5 (4): 37-46.

[16] Chatterjee, D., RajB., Mahata, A., (2001). Adsorption and photocatalytic color removal using fly as hand sunlight. Catalysis Communications, 2: 113-117.

[17] Slokar, Y. M., Lee, A. M., (1998). Marechal Methods of decolorization of textile wastewater. Dyes and Pigm, 37(4): 335-356.

[18] Xia, C., Zhang, J. N., Yang, Z., Guo, S. Y., Guo, S. H., Xu, Q., (2017). 2D MOF nanoflake-assembled spherical microstructures for enhanced supercapacitor and electrocatalysis performance. Nano-Micro Lett, 9(43): 1-11. doi 10.1007/s40820-017-0144-6.

[19] Heydartaemeh, M. R., Aslani, S., Doulati, A. F., (2017). Loess soil nanoparticles as a novel adsorbent for adsorption of green malachite dye. Jr. Chromatogr. Sep. Tech., 8(3): 1-6. doi: 10.4172/2157-7064.1000366.

[20] Chen, J., Chen, W., Zhu, D., (2008). Adsorption of nonionic aromatic compounds to single walled carbon nanotubes: Effect of aqueous solution chemistry. Environmental Sci. and Tech., 42(19): 7225-7230.

[21] Khaled, A., Kapoor, P. N., Klabunde, K. J., (1999). Nanocrystalline metal oxides as new adsorbents for air purification. Nanostructured Materials, 11(4): 459-468.

[22] Sharma, Y. C., Srivastava, V., Mukherjee, A. K., (2010). Synthesis and application of nano $\mathrm{Al} 2 \mathrm{O} 3$ powder for the recalamation of hexavalent chromium from aqueous solutions. Jr. of Chem. and Engg. Data, 55(7): 2390-2398.

[23] Khosla, E., Kaur, S., Dave, P. N., (2013). Mechanistic study of adsorption of Acid Orange-7 over aluminum oxide nanoparticles. Jr. of Engg, 2013. Article ID593534, doi.org/10.1155/2013/593534.

[24] Bautista, P., Mohedano, A. F., Casas, J. A., Zazo, J. A., Rodriguez, J. J., (2008). An overview of the application of Fenton oxidation to industrial waste water treatment. Jr. Chem. Technol. Biotechnol., 83: 1323-1338.

[25] Xu, A., Li, X., Ye, S., Yin, G., Zeng, Q., (2011). Catalyzed oxidative degradation of methylene blue by in situ generated cobalt (II)-bicarbonate complexes with hydrogen peroxide. Appl. Catal. B., 102: 37-43

[26] Wei, W., Gao, P., Xie, J., Zong, S., Cui, H., Yue, X., (2013). Uniform $\mathrm{Cu} 2 \mathrm{Cl}(\mathrm{OH})$ hierarchical microspheres: a novel adsorbent for methylene blue adsorptive removal from aqueous solution. Jr. Sol. State Chem., 204: 305-313. 
[27] Chithambararaj, A. Sanjini, N. S., Bose, A. C., Velmathi, S., (2013). Flower-like hierarchical h-MoO3: new findings of efficient visible light driven nanophotocatalyst for methylene blue degradation. Catal. Sci. Technol., 3:1405-1414.

[28] Chatterjee, D., Rothbart, S., Eldik, van R., (2013). Substrate versus oxidant activation in RuIII (EDTA) catalyzed dye degradation. RSC Adv., 3: 3606-3610.

[29] Etaiw, S. E. H., Saleh, D. I., (2014). The organotin coordination polymer $[(\mathrm{n}-\mathrm{Bu} 3 \mathrm{Sn}) 4 \mathrm{Fe}(\mathrm{CN}) 6 \mathrm{H} 2 \mathrm{O}]$ as effective catalyst towards the oxidative degradation of methylene blue, SpectrochimActa A Mol. BiomolSpectrosc., 117: 54-60.

[30] Le, H. A., Linh, L. T., Chin, S., Jurng, J., (2012). Photocatalytic degradation of methylene blue by a combination of $\mathrm{TiO} 2$-anatase and coconut shell activated carbon. Powder Technol., 225: 167-175.

[31] Impart, O., Katafias, A., Kita, P., Mills, A., PietkiewiczGraczyk, A., Wrzeszcz, G., (2003). Kinetics and mechanism of a fast leuco-methylene blue oxidation bycopper (II)-halide species in acidic aqueous media. Dalton Trans., 3: 348-353.

[32] Varghese, N., Hariharan, M., Cherian, A. B., Sreenivasan, P. V., Paul, J., Antony, A., (2014). PVA - Assisted Synthesis and Characterization of Nano $\alpha$-Alumina. Internat. Jr. of Sci. and Res. Publi., 4(10): 1-5.

[33] Hassena, H., (2016). Photocatalytic degradation of methylene blue by using A12O3/Fe2O3nano composite under visible light. Mod. Chem. Appl., 4: 176. doi:10.4172/23296798.1000176

[34] Byrappa, K., Subramani, A. K., Ananda, S., Lokanatha, R. K. M., Dinesh, R., (2006). Photocatalytic degradation of
Rhodamine B dye using hydrothermally synthesized $\mathrm{ZnO}$. Bull. Mater Sci., 29: 433-438.

[35] Chakrabarti, S., Dutta, B. K., (2004). Photocatalytic degradation of modeltextile dyes in wastewater using $\mathrm{ZnO}$ as semiconductor catalyst. Jr. Hazard Mater., 112: 269-278.

[36] Sun, J., Qiao, L., Sun, S., Wang, G., (2008). Photocatalytic degradation of Orange $\mathrm{G}$ on nitrogen-doped $\mathrm{TiO} 2$ catalysts under visible light andsunlight irradiation. Jr. Hazard Mater., 155: $312-319$

[37] Huang, M., Xu, C., Wu, Z., Huang, Y., Lin, J., (2008). Photocatalytic discolorization of methyl orange solution by $\mathrm{Pt}$ modified TiO2 loaded on natural zeolite. Dyes and Pigm, 77: 327-334.

[38] Wang, C. C., Lee, C. K., Lyu, M. D., Juang, L. C., (2008). Photocatalytic degradation of C. I. Basic Violet 10 using TiO2 catalysts supported by Y zeolite: aninvestigation of the effects of operational parameters. Dyes and Pigm, 76: 817-824.

[39] Macedo, L. C., Zaia, D. A. M., Moore, G. J., Santana, de H., (2007). Degradation of leather dye on TiO2: a study of applied experimental parameters onhotoelectrocatalysis. Jr. of Photochem. andPhotobiol. A: Chemistry, 185 (1): 86-93.

[40] Adar, F., (2014). Molecular spectroscopy workbench Raman spectra of metal oxide. SpectroscopySolutionsfor Materials Analysis. http://www.horiba.com

[41] Banerjee, S., Dubey, S., Gautam, R., Chattopadhyaya, M. C., Sharma, Y. C., (2017). Adsorption characteristics of alumina nanoparticles for the removal of hazardous dye, orange $G$ from aqueous solutions. Arabian Jr. of Chem. http://dx.doi org/j.arabic.2016.12.016 